\title{
PROBLÈMES LIÉS A LA PRODUCTION DES ALLIAGES SUPRACONDUCTEURS A BASE DE NbTi
}

\author{
M. BIDAULT, J. DOSDAT *
}

Thomson C. S. F. Chatou

\begin{abstract}
1. Introduction. - L'étude des phénomènes de supraconductibilité ainsi que la réalisation et l'application des supraconducteurs industriels ont connu, ces huit dernières années, un important essor. Cet essor a été motivé par d'importantes perspectives d'emploi de ces nouveaux matériaux dans de nombreuses branches de la physique moderne. La principale application consiste dans la production de champs magnétiques intenses dans un grand volume. On envisage déjà l'utilisation de ces matériaux en électrotechnique pour la production, le stockage et le transport d'énergie.

Nous allons examiner ici les problèmes posés par la réalisation industrielle de produits supraconducteurs à base d'alliage NbTi.
\end{abstract}

Cette production revêt deux aspects :

- Il faut élaborer un alliage de NbTi présentant :

- Une haute densité de courant critique.

- Une transformabilité aisée.

- Un prix économique.

De plus cet alliage doit être conditionné à l'intérieur d'une matrice assurant la stabilisation, la tenue mécanique et permettant un bobinage aisé.

2. Elaboration de l'alliage NbTi. - La définition de l'alliage $\mathrm{NbTi}$, qui aujourd'hui est produit industriellement, résulte de l'établissement d'un compromis entre des tendances souvent contradictoires. En effet, la haute densité de courant critique recherchée conduit à utiliser un alliage fortement chargé en éléments interstitiels. Au-delà de certaines teneurs cet alliage devient particulièrement difficile à transformer.

L'élément niobium est plus coûteux que le titane. Il importe de réduire au minimum nécessaire la teneur en niobium, mais, dans ce cas, le champ critique $H_{\mathrm{c} 2}$ diminue, comme cela a été montré par ailleurs [1].

Ce compromis nous a donc conduit à choisir deux nuances d'alliage : l'une réservée à la production de champs magnétiques supérieurs à $7 \mathrm{~T}$ et contenant 45 à $40 \%$ de Ti et $60 \%$ de $\mathrm{Nb}$ (champ critique de $12,4 \mathrm{t}$ ), l'autre réservée au domaine des champs infé-

* Actuellement Service Supraconducteurs-THOMSON HOUSTON, Chauny (02). rieurs à $7 \mathrm{~T}$ et contenant $53 \% \mathrm{Ti}$ et $47 \% \mathrm{Nb}$ (champ critique $10 \mathrm{~T}$ ).

L'utilisation d'un niobium non affiné (fortement chargé en tantale) n'apporte que des avantages au point de vue des qualités supraconductrices du produit fini et du prix.

Il apparaît donc que l'établissement des corrélations entre les propriétés supraconductrices de l'alliage et sa composition est extrêmement complexe; nous nous sommes efforcés de définir l'influence déterminante de certains paramètres métallurgiques dont les principaux d'entre eux sont l'écrouissage, les traitements thermiques et la teneur en éléments interstitiels.

Les figures 1, 2, 3 et 4 mettent en valeur l'influence des paramètres fondamentaux.

- La densité de courant critique est améliorée par des taux de déformation élevés (Fig. 1).

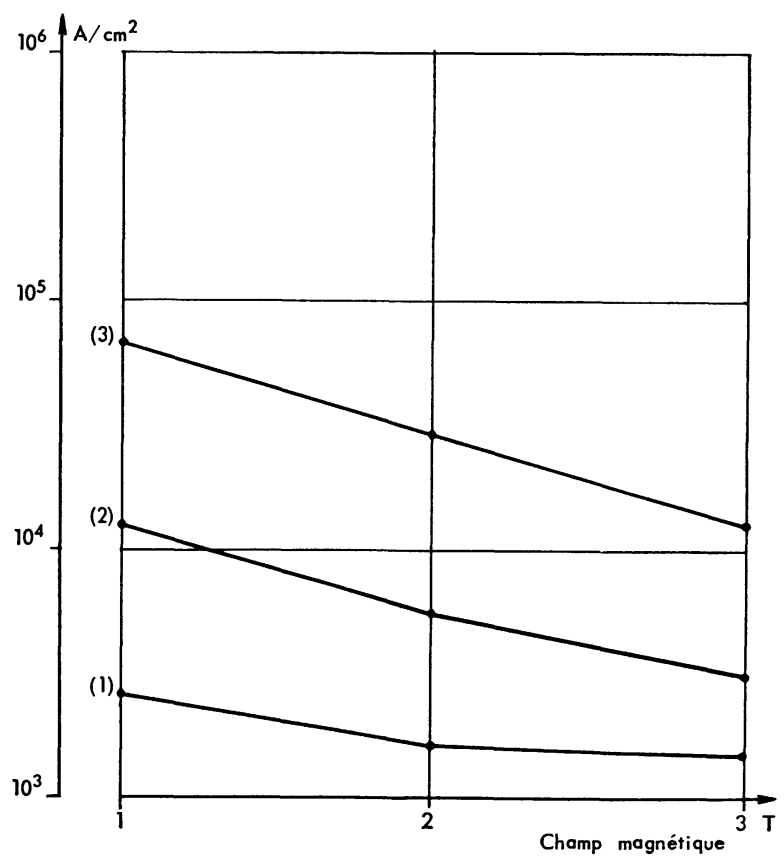

Fig. 1. - Influence des taux de déformation sur le courant critique

1. - Brut de tréfilage (écrouissage $99,8 \%$ ou $87 \%$ ).

2. - Recuit 5 heures à $400{ }^{\circ} \mathrm{C}$ (écrouissage $87 \%$ ).

3. - Recuit 5 heures à $400{ }^{\circ} \mathrm{C}$ (écrouissage $99,8 \%$ ).

$\varnothing 0,25 \mathrm{~mm}$. 
- Les traitements thermiques de l'alliage $\mathrm{NbTi}$ doivent être effectués avec précision, comme le montrent les figures 2 et 3 .

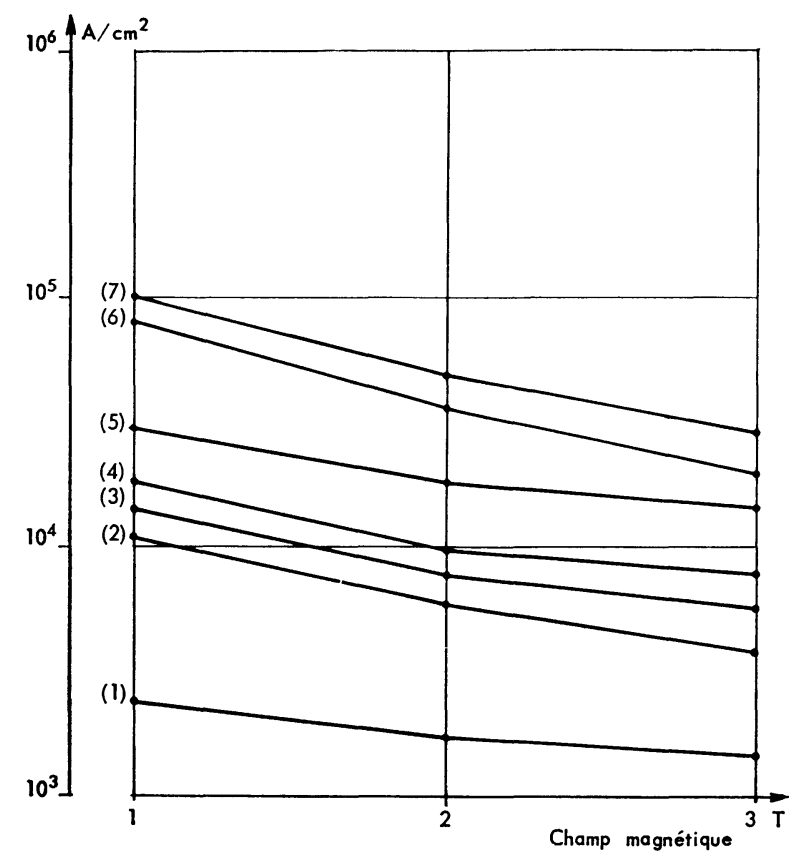

Fig. 2. - Influence de la température du revenu de précipitation sur le courant critique.

1. - Brut de tréfilage.

2. - Recuit 2 heures à $190^{\circ} \mathrm{C}$.

3. - Recuit 15 heures à $190^{\circ} \mathrm{C}$.

4. - Recuit 2 heures à $260^{\circ} \mathrm{C}$.

5. - Recuit 15 heures à $260^{\circ} \mathrm{C}$.

6. - Recuit 15 heures à $430^{\circ} \mathrm{C}$.

7. - Recuit 15 heures à $340^{\circ} \mathrm{C}$.

$\varnothing 0,25 \mathrm{~mm}$.

- La combinaison des traitements thermiques et des traitements mécaniques apporte également une nette amélioration de la densité de courant critique (Fig. 4).

L'ensemble des résultats présentés sur ces figures correspond à une étude préalable de définition des paramètres métallurgiques permettant de comprendre l'incidence de ceux-ci sur les qualités supraconductrices.

L'influence de la teneur en éléments d'insertion a été étudiée sur des alliages semi-industriels et a montré le rôle déterminant du taux en oxygène et en azote sur le courant critique, comme le montre la figure 5 .

Un examen structural plus approfondi a été effectué au microscope électronique sur ces différents matériaux : une corrélation existe entre la densité de courant critique, la teneur en dislocations et l'arrangement des dislocations à l'intérieur du matériau. La figure 6 représente le résultat de l'examen au microscope électronique effectué sur des coupes minces des différents matériaux.

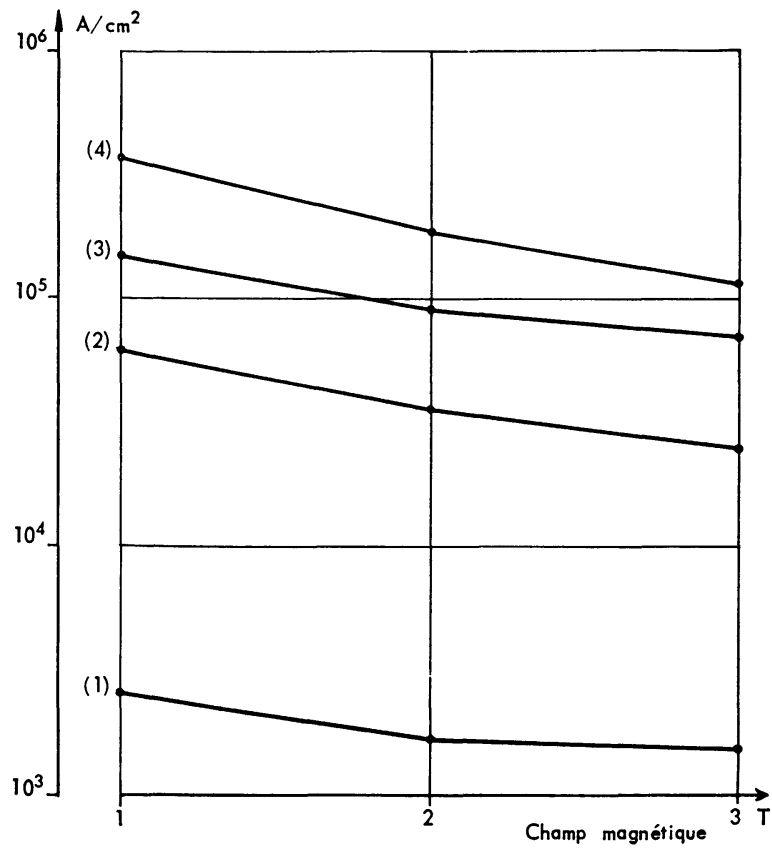

Fig. 3. - Influence de la durée du traitement thermique sur le courant critique.

1. - Brut de tréfilage (non recuit).

2. - Recuit 2 heures à $380^{\circ} \mathrm{C}$.

3. - Recuit 24 heures à $380^{\circ} \mathrm{C}$.

4. - Recuit 85 heures à $380^{\circ} \mathrm{C}$.

$\varnothing 0,25 \mathrm{~mm}$

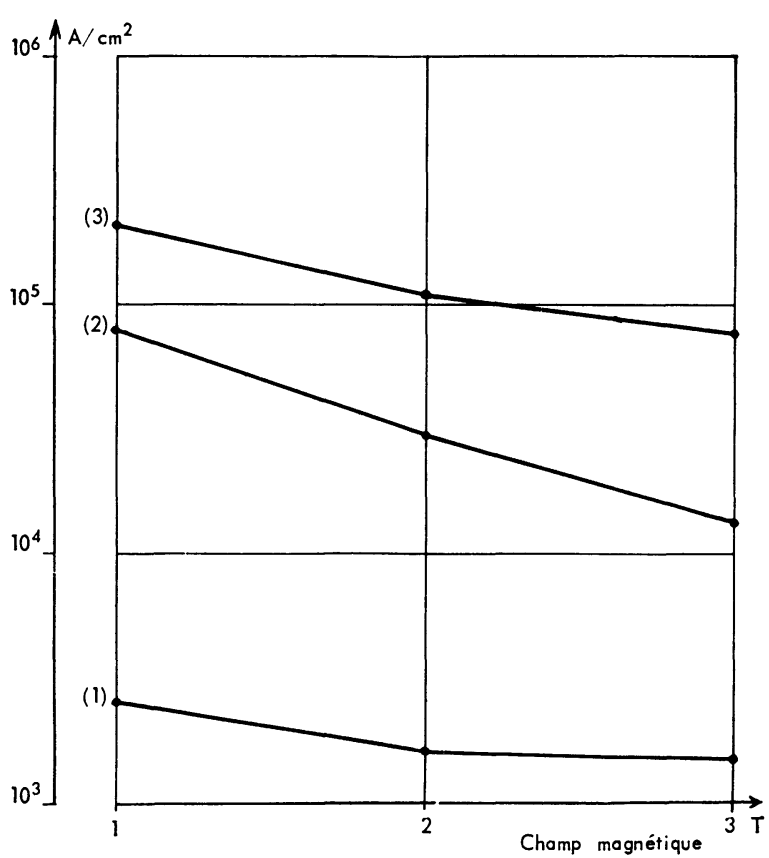

Fig. 4. - Influence des séquences écrouissage-recuit sur le courant critique.

1. - Brut de tréfilage $(\varnothing 0,25 \mathrm{~mm})$.

2. - Brut de tréfilage +5 heures à $400{ }^{\circ} \mathrm{C}(\varnothing 0,25 \mathrm{~mm})$.

3. - Brut de tréfilage +5 heures à $400^{\circ} \mathrm{C}$.

+ tréfilage +5 heures à $400^{\circ} \mathrm{C}$.

+ tréfilage +5 heures à $400{ }^{\circ} \mathrm{C}$.

+ tréfilage +5 heures à $400{ }^{\circ} \mathrm{C}$.

( $0,09 \mathrm{~mm}$ ). 


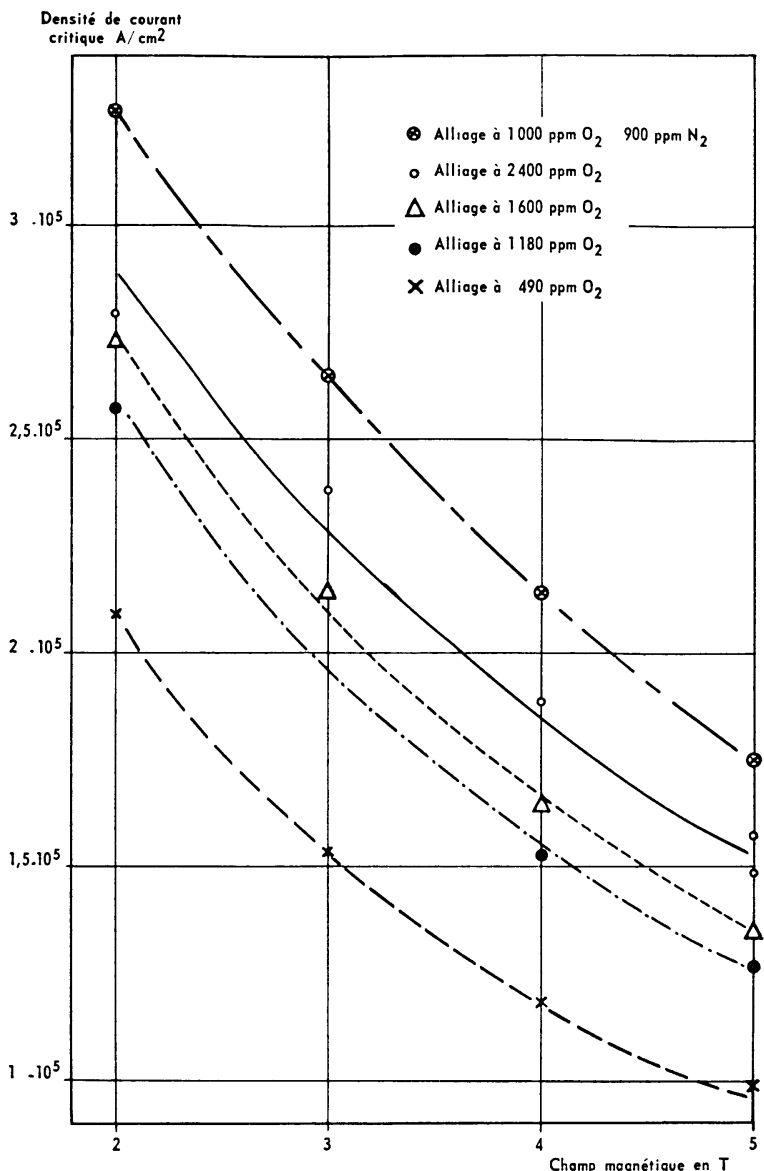

Fig. 5. - Influence de la teneur en oxygène sur la densité de courant critique.

$\otimes$ Alliage à $2400 \mathrm{ppm} \mathrm{O}_{2}$.

$\Delta$ Alliage à $1600 \mathrm{ppm} \mathrm{O}_{2}$.

- Alliage à $1180 \mathrm{ppm} \mathrm{\textrm {O } _ { 2 }}$.

$\times$ Alliage à $490 \mathrm{ppm} \mathrm{O}_{2}$.

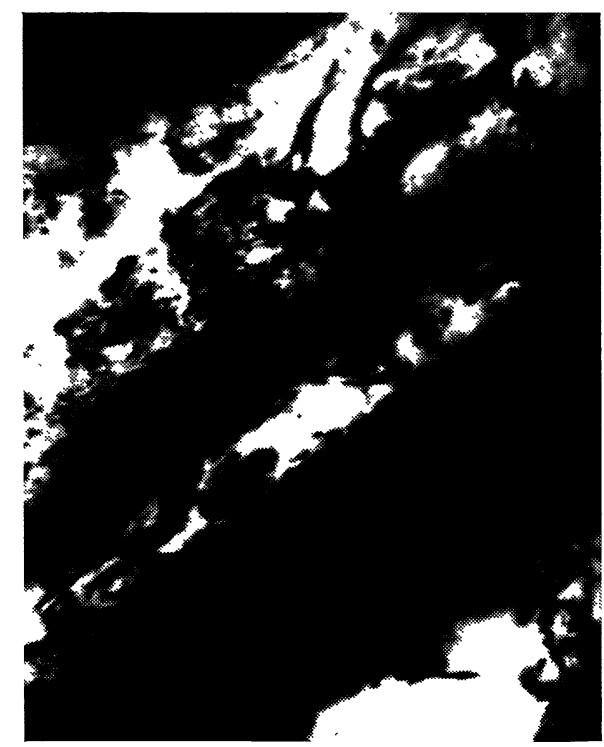

FIG. 6 (a)

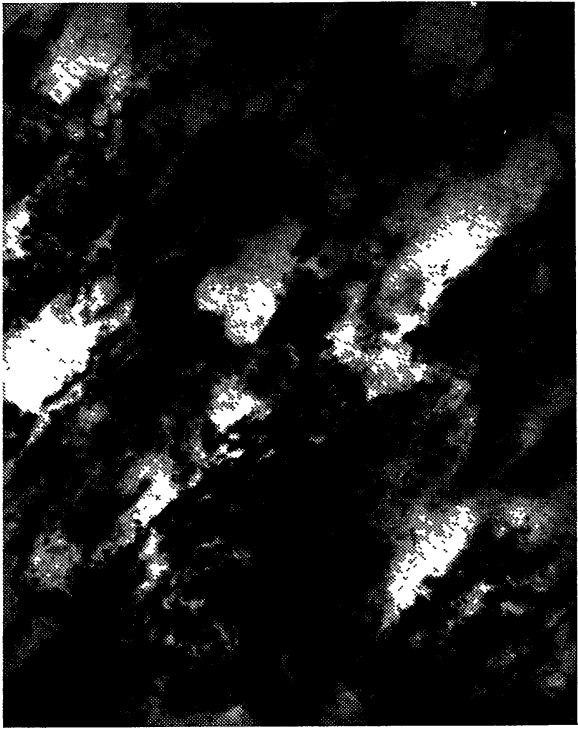

FIG. 6 (b)

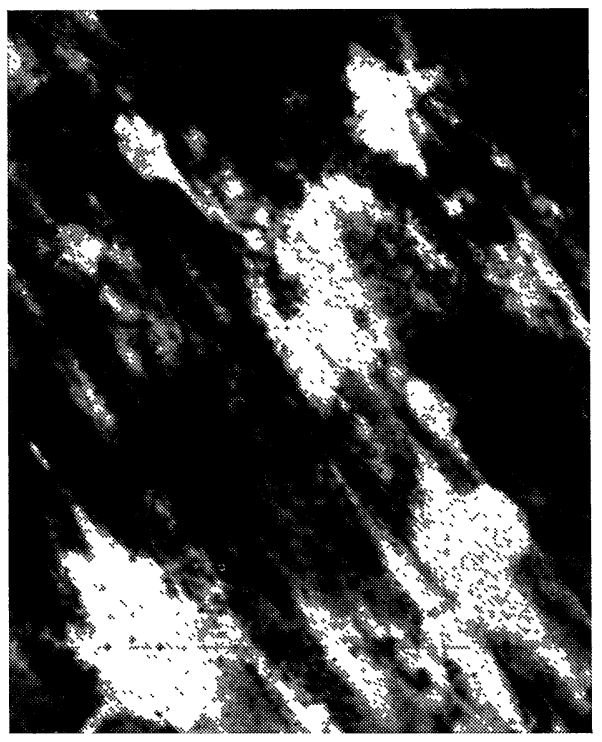

FIG. 6 (c)

FIG. 6. - Influence de la teneur en interstitiels sur la structure de l'alliage NbTi â $40 \%$ en poids de titane.

$a .-490 \mathrm{ppm}$ d'oxygène.

b. $-2400 \mathrm{ppm}$ d'oxygéne.

c. $-1800 \mathrm{ppm}$ d'oxygène $+966 \mathrm{ppm}$ d'azote.

Lames minces examinées au microscope électronique. $G: 60000$.

Le tableau I résume les principales relations existant entre les aspects micrographiques de chaque alliage et la densité de courant critique à 5 Tesla.

Des recherches d'alliage du type ternaire ont également été poursuivies sans que des avantages marquants par rapport à l'alliage binaire $\mathrm{NbTi}$ aient été mis en valeur. Les compositions NbTiZr, NbTiHf, NbTiTa, NbTiV présentent des valeurs plus faibles du champ critique, sans pour autant conduire à une densité de courant critique améliorée.

Ces différentes études ont été conduites grâce à l'appui de la DGRST Comité Métallurgie. 
Tableau I

\begin{tabular}{|c|c|c|c|}
\hline Alliage & Alliage dopé à 490 ppm $\mathrm{O}_{2}$ & Alliage dopé à 2400 ppm $\mathrm{O}_{2}$ & $\begin{array}{c}\text { Alliage dopé à } 1870 \mathrm{ppm} \mathrm{O}_{2} \\
\text { et } 966 \mathrm{ppm} \mathrm{N} \mathrm{N}_{2}\end{array}$ \\
\hline \multirow[t]{3}{*}{$\overline{\text { Microstructure }}$} & $\begin{array}{l}\text { 1) bandes de largeur } 0,1 \text { à } \\
0,2 \mu\end{array}$ & $\begin{array}{l}\text { 1) bandes filiformes de lar- } \\
\text { geur } 0,03 \text { à } 0,1 \mu\end{array}$ & $\begin{array}{l}\text { 1) bandes lenticulaires fines } \\
\text { de largeur } 0,05 \mu \text { à } 0,1 \mu\end{array}$ \\
\hline & $\begin{array}{l}\text { 2) structure interne ponctuée } \\
\text { peu chargée en défauts fins }\end{array}$ & $\begin{array}{l}\text { 2) structure interne finement } \\
\text { ponctuée } \varnothing 0,01 \text { et } 0,03 \mu\end{array}$ & $\begin{array}{l}\text { 2) structure interne à fin } \\
\text { réseau cellulaire de maille } \\
\text { inférieure à } 0,01 \mu\end{array}$ \\
\hline & $\begin{array}{l}\text { 3) pas de précipitation fine } \\
\text { visible }\end{array}$ & 3) globules de $\varnothing 0,01$ à $0,03 \mu$ & 3) pas de précipitation visible \\
\hline $\begin{array}{l}\text { Densité de cou- } \\
\text { rant critique à } \\
5 \text { Tesla }\end{array}$ & $1 \times 10^{5} \mathrm{~A} / \mathrm{cm}^{2}$ & $1,5 \times 10^{5} \mathrm{~A} / \mathrm{cm}^{2}$ & $1,7 \times 10^{5} \mathrm{~A}$ \\
\hline
\end{tabular}

Ces études nous ont conduits à la définition d'un alliage dont les opérations de transformation industrielle comportent les grandes étapes suivantes :

- La production de billettes, d'un poids unitaire de 50 à $100 \mathrm{~kg}$, est réalisée au four à arc à électrode consommable. Deux fusions permettent d'obtenir l'homogénéité structurale requise.

- Un forgeage à chaud suivi de martelage, laminage, étirage à froid permet d'aboutir à un semi-produit utilisable dans les diverses opérations de conditionnement (rond de 7 à $10 \mathrm{~mm}$ de diamètre).

3. Conditionnement. - Comme l'ont montré de nombreuses études, le niobium titane doit être stabilisé par un matériau à très faible résistivité [2]. Une solution intéressante consiste alors à noyer les filaments de NbTi dans une matrice de cuivre ou d'aluminium. Cette matrice est élaborée dans une géométrie convenable pour le type de bobinage envisagé ce qui conduit à des conducteurs composites en forme de fil, ruban, tube.

En fonctionnement lors d'une transition accidentelle du matériau de l'état supraconducteur à l'état normal, elle constitue un shunt électrique et protège le supraconducteur contre un emballement du blocage sur toute la longueur.

Cette matrice présente également une grande conductibilité thermique ce qui permet d'abaisser toute la masse du conducteur composite à celle de l'hélium liquide réfrigérant.

Pour bénéficier pleinement des avantages apportés par cette matrice de cuivre ou d'aluminium, le constructeur doit s'efforcer de satisfaire trois conditions :

- Il doit choisir une matrice à très faible résistivité électrique : le cuivre de haute pureté apporte une solution intéressante puisqu'il est possible de trouver à échelle industrielle des cuivres présentant une résistivité de

$$
\begin{aligned}
& R_{0}=10^{-8} \Omega / \mathrm{cm} \text { sous induction nulle. } \\
& R_{0}=2,5 \text { à } 3,5 \times 10^{-8} \Omega / \mathrm{cm} \text { sous induction de } 5,5 \mathrm{~T} .
\end{aligned}
$$

L'aluminium A 99 est également intéressant puisqu'il présente à $4,2{ }^{\circ} \mathrm{K}$ une résistivité de $\rho=0,2$ à $0,3 \times 10^{-8} \Omega / \mathrm{cm}$ sous induction nulle. Son absence de magnétorésistance à partir de $20 \mathrm{kG}$ peut le faire préférer dans certaines applications.

- Il doit assurer une liaison de type métallurgique entre le cuivre et le NbTi de manière que, au cours d'un blocage du supraconducteur, le courant soit dérivé dans le cuivre avec des pertes joules minimales. Cette liaison est réalisée par différentes techniques de transformation, Thomson-Brandt a breveté divers procédés de coétirage et de colaminage. Avec un matériau à faible résistivité placé en quantité suffisante et une liaison métallurgique parfaite, il est ainsi possible d'obtenir un fonctionnement réversible du matériau comme le montre la figure 7 .

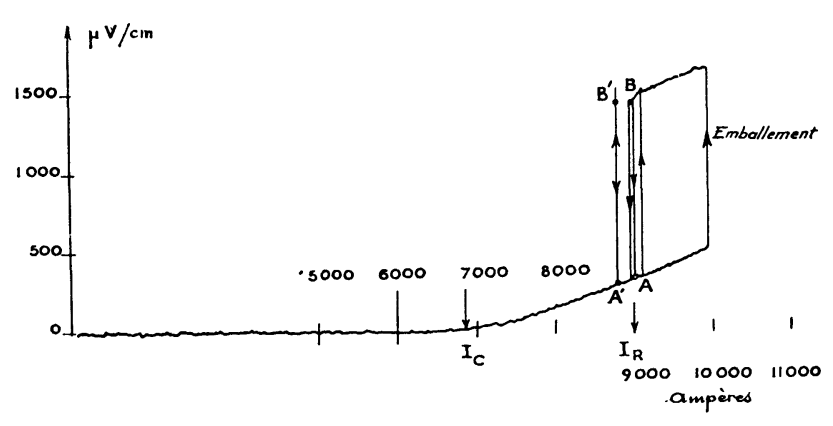

FIG. 7. $-I_{\mathrm{a}}$ : intensité critique.

$I_{R}:$ intensité de récupération.

Placé dans un champ donné et traversé par un courant supérieur à sa valeur critique, point $\mathrm{A}$, l'échantillon est sollicité par une impulsion thermique qui bloque le matériau, tout le courant passe alors dans le cuivre, point B. A la fin de l'impulsion thermique le matériau retrouve l'état supraconducteur point $\mathrm{A}$.

- Pour les réalisations de grands bobinages, il est souvent nécessaire de confier à la matrice un rôle supplémentaire : elle doit aussi assurer la tenue méca- 
nique. Le cuivre présente dans ce cas un avantage majeur sur l'aluminium de haute pureté.

Module d'élasticité du cuivre ....... $11 \mathrm{~kg} / \mathrm{mm}^{2}$ Module d'élasticité de l'aluminium .... $3 \mathrm{~kg} / \mathrm{mm}^{2}$

La courbe de la figure 8 montre pour le cuivre une étroite corrélation entre l'allongement sous contrainte et la résistivité électrique, la dureté étant en paramètre, en particulier il est possible d'utiliser des cuivres travaillant à des taux de contrainte de l'ordre de $10 \mathrm{~kg} / \mathrm{mm}^{2}$ et présentant une résistivité meilleure que $3 \times 10^{-8} \Omega / \mathrm{cm}$ sans induction.

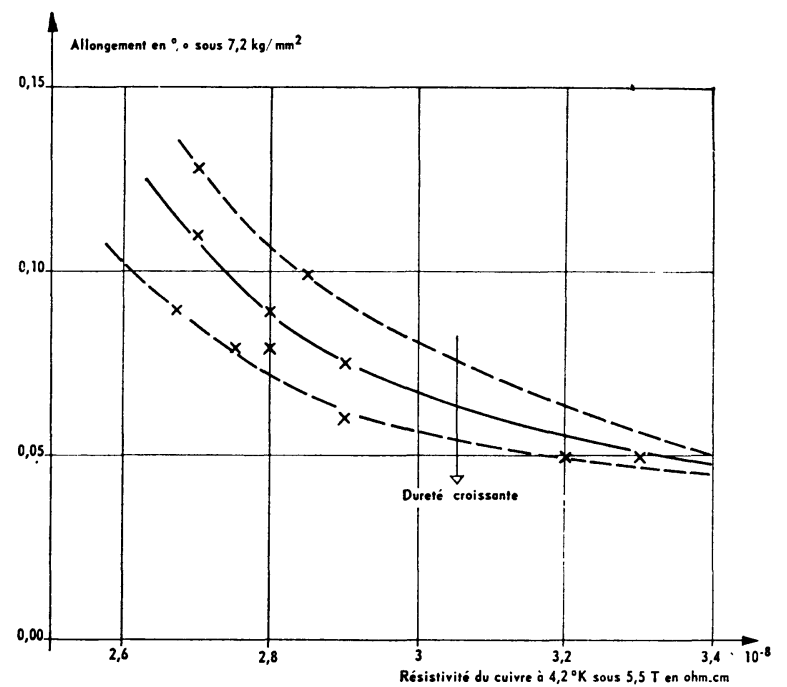

Fig. 8. - Relation entre l'allongement $\%$ sous $7,2 \mathrm{~kg} / \mathrm{mm}^{2}$ et la résistivité du cuivre à $4,2^{\circ} \mathrm{K}$ sous $5,5 \mathrm{~T}$.

Quelques exemples de réalisation peuvent être cités :

- Le CENS de Saclay a réalisé un bobinage de $1 \mathrm{~m}$ de diamètre intérieur et produisant un champ de $40 \mathrm{kG}$ au centre pour une énergie stockée de 10 mégajoules. Le ruban utilisé transporte $1500 \mathrm{~A}$ à $5,5 \mathrm{~T}$.

- Le ruban, dont la photo est donnée figure 9, est prévu pour équiper la grande chambre à bulles

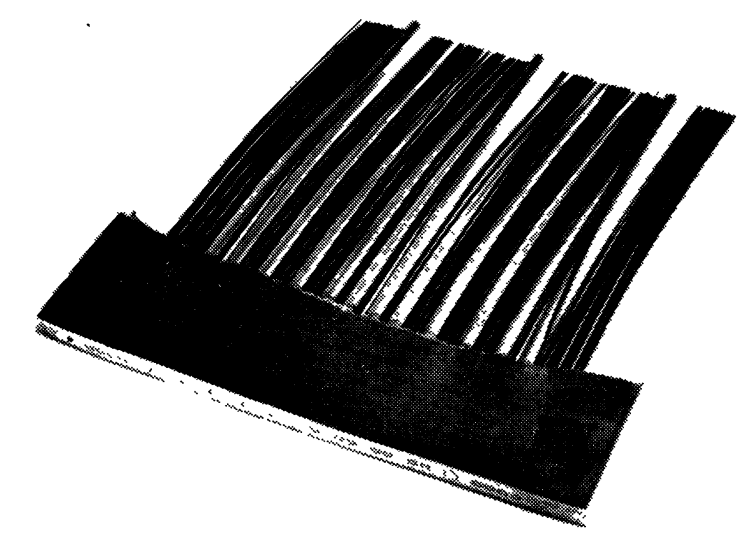

FIG. 9. - Ruban composite homothétique du ruban devant équiper la chambre à bulles du CERN. On remarque sur la partie décuivrée les 220 fils supraconducteurs de $0,2 \mathrm{~mm}$ de diamètre et les trois fils de renfort mécanique en acier inoxydable. européenne qui sera construite au CERN. 21 longueurs unitaires de ce ruban doivent être produites et correspondront après assemblage à un pôle de l'aimant de la chambre à bulles. L'intensité nominale prévue est de 5700 A pour des courants critique et reversible respectivement de 6450 et $7000 \mathrm{~A}$ à $4,5{ }^{\circ} \mathrm{K}$.

Il est à noter que ce matériau est produit en longueur unitaire de $1500 \mathrm{~m}$ ce qui représente, un poids total de cuivre de $2400 \mathrm{~kg}$ et un poids de $\mathrm{NbTi}$ de $80 \mathrm{~kg}$.

Dans le cas particulier de cette chambre à bulles, l'énergie stockée est l'ordre de 800 mégajoules et des coefficients de sécurité importants ont été pris pour assurer une très bonne stabilisation du matériau, ce qui explique la grande proportion de cuivre utilisée

$$
\frac{\text { poids du cuivre }}{\text { poids de NbTi }}=30 \text {. }
$$

- Le refroidissement des matériaux supraconducteurs, par simple immersion dans l'hélium liquide à pression normale, limite le coefficient d'échange thermique à une valeur de $0,25 \mathrm{~W} / \mathrm{cm}^{2}$. L'utilisation de l'hélium supercritique circulant sous pression, à l'intérieur de conducteur composite creux, permet d'améliorer le coefficient d'échange thermique. Ce procédé simplifie par ailleurs le cryostat et laisse la possibilité de placer une isolation électrique importante sur le conducteur sans réduire son refroidissement. La figure 10 donne un exemple de réalisation d'un tel produit.

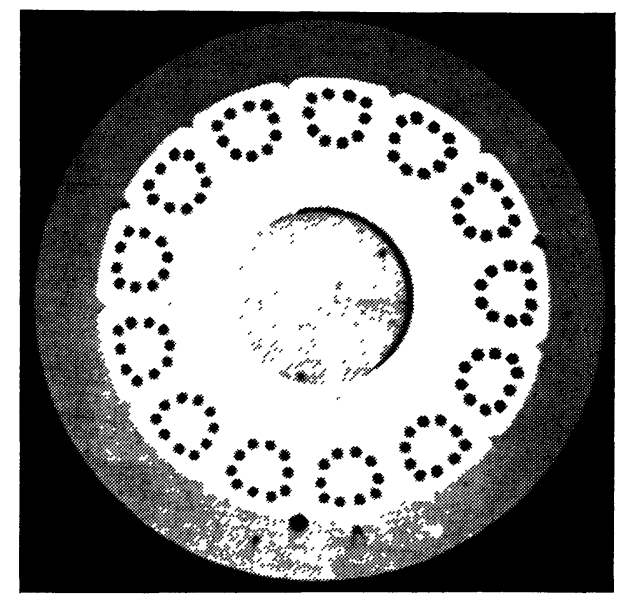

Fig. 10. - Conducteur creux à 130 filaments de diamètre $150 \mu$.

- Les progrès technologiques d'une part et les études théoriques d'autre part, poursuivis en parallèle, ont montré qu'il y avait un grand intérêt à utiliser des filaments fins de manière à obtenir une stabilisation intrinsèque du supraconducteur, la quantité de cuivre nécessaire étant réduite à des proportions plus faibles

$$
\frac{\text { poids cuivre }}{\text { poids supra }} \sim 1
$$


Pour réaliser des conditions de stabilisation intrinsèques, la taille des filaments d'alliage $\mathrm{NbTi}$ doit être inférieure à $50 \mu$ [2].

La figure 11 donne la coupe micrographique $\mathrm{du}$ matériau à stabilisation intrinsèque, développé par Thomson, comportant 703 filaments de $1,5 \mu \mathrm{de}$ diamètre ; la densité de courant critique dans l'alliage $\mathrm{NbTi}$ seul est de $1,6 \times 10^{5} \mathrm{~A} / \mathrm{cm}^{2}$ à $5 \mathrm{~T}$.

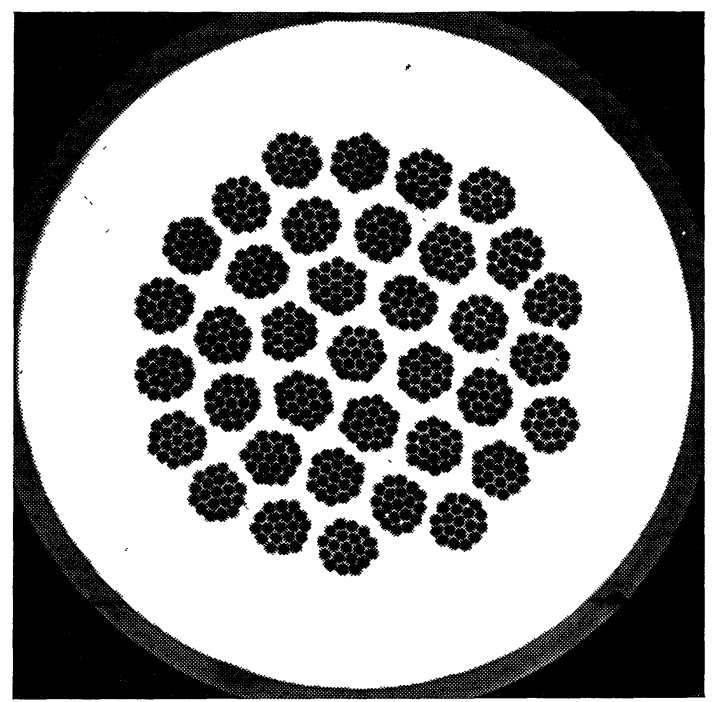

FIG. 11. - Conducteur multifilamentaire à 703 filaments de diamètre $1,5 \mu$.

La densité apparente de ce type de matériau est fonction, naturellement, de la quantité de cuivre ; elle peut atteindre $40000 \mathrm{~A} / \mathrm{cm}^{2}$ à $5 \mathrm{~T}$.

Il est à noter qu'un tel conducteur présente un courant reversible égal ou supérieur au courant critique pour une valeur minimum du champ magnétique de $3 \mathrm{~T}$.

Ces produits composites à filaments fins constituent une approche intéressante pour les applications des supraconducteurs en champ variable.

De nombreuses études théoriques et expérimentales [3 et 4] sont poursuivies sur ce sujet ; elles ont montré qu'il était nécessaire, d'une part de réduire le diamètre des filaments à des tailles de l'ordre du micron et, d'autre part, de transposer les filaments à l'intérieur de la matrice qui doit être résistive (alliage de cuivre).

Ces deux critères essentiels ont été étudiés. L'ensemble des résultats théoriques est donné ci-après :

1. - Dans un filament simple il n'y aura pas de saut de flux si la condition suivante est satisfaite :

$$
d \leqslant\left(10^{9} S T_{0} f\right)^{1 / 2} / J_{\mathrm{c}}
$$

avec $S$ chaleur spécifique (Joules $/ \mathrm{cm}^{3}{ }^{\circ} \mathrm{K}$ )

$$
T_{0}\left({ }^{\circ} \mathrm{K}\right)=J_{\mathrm{c}}\left(f \mathrm{~d} J_{\mathrm{c}} / \mathrm{d} T\right) \simeq T_{\mathrm{c}} / 2 .
$$

$f$ facteur numérique de l'ordre de 0,9 . Avec une densité de courant à champ faible : $J_{\mathrm{c}}=3 \times 10^{5} \mathrm{~A} /$ $\mathrm{cm}^{2}$ on obtient typiquement;

$$
d \text { (diamètre du filament) } \leqslant 0,05 \mathrm{~mm} \text {. }
$$

2. - Dans un matériau composite, multifilamentaire, on peut définir une longueur critique $l_{\mathrm{c}}$ :

$$
l_{c}^{2} \simeq 10^{8} \lambda J_{\mathrm{c}} d \rho / \dot{H}
$$

avec $\lambda$ facteur spatial de forme,

$J_{\text {c }}$ densité de courant,

$d$ épaisseur de supraconducteur,

$\rho$ résistivité de la matrice,

$\dot{H}$ vitesse de variation du champ magnétique.

$l_{\mathrm{c}}$ est typiquement de l'ordre de quelques centimètres avec une matrice en cuivre. Si les filaments de diamètre $d$ sont placés parallèlement dans la matrice, le conducteur se comporte comme un filament simple de diamètre $D$, diamètre total du conducteur.

Si par contre on effectue un toronage des filaments avec un pas inférieur à $l_{\mathrm{c}}$, seul intervient le diamètre $d$.

En conclusion, pour un matériau donné, les paramètres importants sont :

- Le diamètre des filaments supraconducteurs.

- Le pas de transposition.

- La résistivité de la matrice.

- La densité de courant critique.

- Le rapport des volumes de la matrice et du supraconducteur.

Le cliché de la figure 12 montre l'aspect d'un composite transposé.

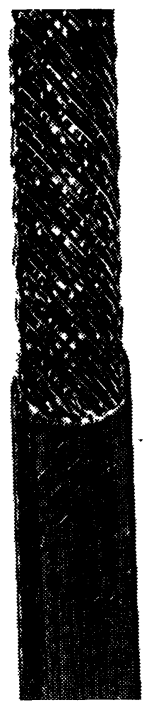

Fig. 12. - Rubans multifilamentaires twistés.

Un large programme de mesure des pertes en champ variable produit par des matériaux de ce type est actuellement en cours de réalisation.

L'effort technologique à poursuivre portera essentiellement sur la diminution du diamètre utile des filaments et sur l'emploi de nouvelles matrices isolantes, voir composites, de manière à optimiser les paramètres tels que :

- Résistance électrique.

- Isolation électrique.

- Conductibilité thermique.

- Tenue mécanique. 
4. Conclusion. - Depuis plusieurs années l'emploi systématique de fils composites a permis de mettre à la disposition des chercheurs des bobinages à champs intenses et homogènes $\left(6 \mathrm{~T}\right.$ et $\Delta B / B \sim 10^{-6} / \mathrm{cm}^{3}$ ) leur offrant ainsi un domaine d'expérimentation que les électro-aimants classiques ne permettaient pas d'explorer jusqu'alors.

Aujourd'hui la production de la gamme des matériaux nécessaires à la génération de champs magnétiques intenses dans les forts volumes atteint le stade industriel comme le montrent les importantes réalisations en cours dans le domaine de la physique des hautes énergies. On peut citer entre autres:

- La réalisation BIM du CENS Saclay (4 T, diamètre utile $1 \mathrm{~m}, 10$ mégajoules).

- Le bobinage pour les chambres à bulles d'Argonne, de Brookhaven et du CERN.
Dans ce dernier cas :

$$
\begin{array}{ll}
B \ldots \ldots \ldots \ldots \ldots \ldots \ldots & 3,5 \mathrm{~T} \\
\text { Diamètre } \ldots \ldots \ldots \ldots \ldots & 4,6 \mathrm{~m} \\
\text { Energie stockée } \ldots \ldots \ldots \ldots & 800 \text { mégajoules }
\end{array}
$$

Malgré les résultats incomplets dont on dispose actuellement quant à l'importance des pertes en champ variable, on peut espérer l'utilisation de certains de ces matériaux pour les réalisations d'aimants de synchrotron dans un avenir relativement proche.

L'utilisation systématique des supraconducteurs dans les grands domaines de l'électrotechnique implique encore d'importants travaux d'étude et de développement, aussi bien sur les matériaux que sur la cryogénie, mais elle assurera vraisemblablement une transformation marquante des techniques de généiation, de transport et de stockage de l'énergie électrique.

\section{Bibliographie}

[1] Berlincourt, Phys. Rev., 1963, 131, 140.

[2] LAVERICK (C.), Progress in the development of superconducting magnets, Cryogenics, 1965, 152.

LAVERICK (C.) and LobeLL (G.), Large high field superconducting magnet system, Rev. Sci. Instr., 1965, 36, 825 .
[3] SMith (P. F.), Wilson (M. N.), Walters (C. R.), LERVIN (J. D.), Intrinsically stable conductors. A publier : proceedings of 1968 Summer Study on Superconducting Devices and Accelerator (Brookhaven National Laboratory). 